\title{
Fine Dispersion and Property Differentiation of Nanoscale Silicate Platelets and Spheres in Epoxy Nanocomposites
}

\author{
Chien-Chia CHU, ${ }^{1}$ Jiang-Jen LIN, ${ }^{1, \dagger}$ Chang-Ru SHIU, ${ }^{1}$ and Chang-Chin KwAN ${ }^{2, \dagger}$ \\ ${ }^{1}$ Department of Chemical Engineering, National Chung Hsing University, Taichung, Taiwan \\ ${ }^{2}$ Department of Applied Chemistry, Providence University, Taichung, Taiwan
}

(Received August 20, 2004; Accepted January 4, 2005; Published April 15, 2005)

\begin{abstract}
Silica spheres (with an averaged diameter of $10 \mathrm{~nm}$ ) and silicate platelets (approximately $100 \times$ $100 \times 1 \mathrm{~nm}^{3}$ in dimension) were allowed to disperse in polyoxypropylene-triamine $(400 \mathrm{~g} / \mathrm{mol}$ molecular weight), then cured with the epoxy resin diglycidyl ether of bisphenol-A (DGEBA). With 1-5 wt \% loading of these inorganic silicates, the cured epoxies exhibited high hardness, transparency, and a low thermal expansion coefficient. These silicate platelets also enhance the epoxy hardness from the pristine $2 \mathrm{H}$ to $4 \mathrm{H}$ while adding only $0.5 \mathrm{wt} \%$. By comparison, if the spherical silica is used, a similar hardness can only be achieved by loading as high as $5 \mathrm{wt} \%$ of the silica. The high aspect-ratio and fine dispersion of the platelet silicates were found to be important factors in influencing the cured epoxy's properties. In addition, a TEM micrograph shows that the silicate platelets are well-dispersed and have a unique self-arranged lamellar orientation. [DOI 10.1295/polymj.37.239]

KEY WORDS Silicate Platelets / Montmorillonite / Exfoliation / Silica Oxide / Epoxy /
\end{abstract}

The fine dispersion of nanoscale inorganic particles in organic polymers has proven to be feasible for promoting favorable material properties and increasing the effectiveness of their practical applications. ${ }^{1-3}$ Both silica oxide spheres and layered silicate clays are known for advancing the physical properties of nanocomposites. ${ }^{4-10}$ In general, spherical silica oxides are synthetic while the layered silicates are obtained from the naturally-occurring clays. ${ }^{11}$ Extensive studies have focused on the utilization of the layered structures as additives in organic polymers to enhance properties such as mechanical strength, ${ }^{12-16}$ thermostability, ${ }^{17,18}$ chemical resistance, ${ }^{19,20}$ gas barrier properties, ${ }^{21}$ solvent resistance, ${ }^{20}$ etc. In order to mix with highly hydrophobic polymers, these silicate clays and silicate oxides require organic modification to overcome their inherently hydrophilic nature and lack of affinity for the hydrophobic organic polymers. Homogeneous dispersion is therefore the main issue for preparing these nanocomposites.

While the silica oxide particles can be successfully surface-modified, the layered clays are multiple silicate layers and require an organic intercalation into the layered galleries. The intercalation of layered silicates is typically performed through an ionic exchange reaction. For example, the sodium montmorillonite can be intercalated by low-molecular-weight alkyl quaternary ammonium salts. As a result, the basal spacing of layered silicates is enlarged to about 20-30 A. ${ }^{1-6}$ The resulting organic encapsulation often increased the compatibility of the clays and, in some cases, increase hydrophobicity enough to prepare nanocomposites. Recently, we have reported the use of high-molecular-weight poly(oxypropylene)diamine (POP-amine) as intercalating agents of the organoclay to achieve a wide interlayer spacing organoclay of up to $92 \AA^{22}$ and increased compatibility with epoxy resin. Subsequently, it was found that a tailored POP-amine derivative using a Mannich reaction with formaldehyde and $p$-cresol was suitable for direct clay exfoliation into random silicate platelets. After aqueous $\mathrm{NaOH}$ extraction, the silicate platelet suspension in water was obtained at a $2 \mathrm{wt} \%$ concentration. The individual platelets with sodium counter ions have an average dimension $100 \times 100 \times 1 \mathrm{~nm}^{3}$. The exfoliated silicate platelets resemble the silicate oxide in composition except for their geometric shape and surface ionic characteristics.

The unique plate-like morphology and high aspectratio prompted us to investigate its difference from the common spherical silicate oxide with respect to their physical properties. In this paper, we compare the geometric shape parameter between the platelet and the spherical silicates when interacting with epoxy polymers. The differences in aspect-ratio and dispersion of the mixed nanoscale silicates in the polymer matrix may play an important role in affecting the composite properties. The epoxy system of diglycidyl ether of bisphenol-A (DGEBA) curing with polyoxyalkylene-triamines (Jeffamine ${ }^{\circledR}$ Amine T403) has been chosen for the comparison. The resultant physical properties are further correlated with the silicate

${ }^{\dagger}$ To whom correspondence should be addressed (Tel: +886-4-2285-2591, Fax: +886-4-2287-1787, E-mail: JJLin@dragon.nchu.edu.tw). 
(a)<smiles>CCC(=O)CC(CC(=O)OCC(C)N)CC(=O)C(C)N</smiles>

Jeffamine $^{\circledR}$ T403 a=0.5 1

(b)

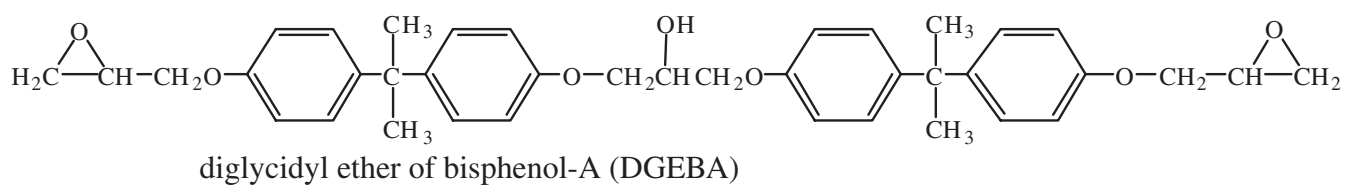

Figure 1. Chemical structures of (a) trimethyol-propane initiated poly(propylene glycol)-tri(2-aminopropyl ether) at $M_{\mathrm{w}}$ of 400 (Jeffamine $^{\circledR}$ T403) (b) liquid diglycidyl ether of bisphenol-A (DGEBA).

platelet dispersion morphology observed in transmission electron microscopy.

\section{EXPERIMENTAL}

\section{Materials}

Sodium montmorillonite $\left(\mathrm{Na}^{+}\right.$-MMT), a smectite clay with a cation exchange capacity (CEC) of 1.20 mequiv/g, was supplied by Nanocor Co. Liquid diglycidyl ether of bisphenol-A (DGEBA, trade name BE188) with an epoxide equivalent weight (EEW) of 188 was obtained from Nan-Ya Chemicals (Taiwan). Trimethyol-propane initiated poly(propylene glycol)tri(2-aminopropyl ether) at $M_{\mathrm{w}}$ of 400 (Jeffamine $^{\circledR}$ T403) (Figure 1) was purchased from Huntsman Chemical Co. The $10 \mathrm{~nm}$ silica oxide $(15 \mathrm{wt} \%$ in water) was supplied by Chang Chun Plastics Co.

\section{Preparation of Random Silicate Platelets}

Random silicate platelets are prepared from layered $\mathrm{Na}^{+}$-MMT according to the procedures developed in our laboratories and will be reported elsewhere. ${ }^{23}$ Typically, the direct exfoliation process was performed by using a polymeric Mannich amine as the exfoliating agent through ionic exchange reaction. The water suspended, exfoliated random silicate platelets was isolated by a toluene/water two-phase extraction to remove the amine exfoliating agent. The silicate platelets were characterized by a laser particle size analyzer to have an average diameter of $c a$.
$100 \mathrm{~nm}$, in contrast to the pristine $\mathrm{Na}^{+}-\mathrm{MMT}$ $850 \mathrm{~nm}$ aggregates. An up to $2 \mathrm{wt} \%$ silicate platelet suspension in water was obtained. The random silicate platelets in irregular polygon shapes are estimated to have an area dimension of approximately $100 \mathrm{~nm}$.

Converting of Nanoscale Silicate Particles and Platelets in Water into T403 Amine Curing Agent Medium

The typical process for converting the silicate/ water suspension into amine medium is described below. A $2 \mathrm{wt} \%$ silicate platelet (or $15 \mathrm{wt} \%$ silica oxide) in water suspension ( $100 \mathrm{~g})$ was added with Jeffamine ${ }^{\circledR}$ T403 $(98 \mathrm{~g})$ in a round-bottomed flask, which was subjected to a rotary evaporator under a reduced pressure at $60^{\circ} \mathrm{C}$ to remove water until dryness. The resultant suspension was clear, transparent, and viscous medium.

\section{Curing Process}

Silicate platelets and silica oxide particles of different weight fractions $(0.05-0.5 \mathrm{~g})$ in the Jeffamine ${ }^{\circledR}$ T403 curing amine $(2.63 \mathrm{~g}, 6.5 \mathrm{mmoles})$ were mixed with a designated amount of DGEBA $(7.37 \mathrm{~g}, 20$ mmoles), on an equal equivalent ratio of epoxide/ $\mathrm{NH}$. The mixture was thoroughly stirred, degassed and poured into a $1 / 8$-inch-thick flat aluminum mold to form a sample plaque or spin-coated on copper surface form a film. The curing process was conducted in an oven at programmed temperatures of $80^{\circ} \mathrm{C}$ for $2 \mathrm{~h}$, $120^{\circ} \mathrm{C}$ for $2 \mathrm{~h}$, and $150^{\circ} \mathrm{C}$ for $1 \mathrm{~h}$. 


\section{Characterization}

The X-ray diffraction (XRD) was recorded on a Shimadzu SD-D1 diffractometer with $\mathrm{Cu}$ target $(k=$ $1.5405 \AA)$. The basal spacing $(n=1)$ was calculated according to Bragg's equation $(n \lambda=2 d \sin \theta)$ through the observed peaks of $n=2,3$, etc. Thermal gravimetric analysis (TGA) was performed on a Seiko SII model SSC/5200, by heating the samples from 50 to $850^{\circ} \mathrm{C}$ at $10^{\circ} \mathrm{C} / \mathrm{min}$ in nitrogen. Thermal mechanical analyzer (TMA) was performed on a Du Pont Instrument-TMA2940, A heating or cooling rate of $10{ }^{\circ} \mathrm{C} / \mathrm{min}$ under nitrogen atmosphere was used for $T_{\mathrm{g}}$. Transmission electronic microscopy (TEM) was performed on a Zeiss EM 902A and operated at 120 $\mathrm{kV}$. The sample was prepared by encapsulation in epoxy material. Film transparency is measured by using an UV-vis absorption at $550 \mathrm{~nm}$ on a PerkinElmer Lambda 20 UV-vis spectrophotometer. Pencil Hardness was measured according to the method of ASTM D 3363-74. The fractured surfaces were carried out on a scanning electron microscopy (SEM) (A JEOL JSM5600 scanning electronic microscope, operated at $15 \mathrm{kV})$.

\section{RESULTS AND DISCUSSION}

\section{Geometric Shape Difference between Silicate Plate-} lets and Spherical Particles

The spherical silicate oxide is commonly used as an inorganic additive to reinforce epoxy properties such as hardness and thermal stability. Similarly, the layered silicate clays have been organically intercalated and dispersed into epoxy nanocomposites. Their geometric shape differences, in particular, the aspect ratio (surface area to thickness), cause the interaction of their surface areas with the surrounded organic matrices to be significantly dissimilar. The surface area for a spherical particle is proportional to the radius equation of $4 \pi r^{2}$, while the surface area of square platelet shape, with its thin thickness $(T)$, is closely related to its platelet area, $2 L^{2}$. Assuming an equal density, the dimensions of the different shapes may correlate by the equation, $L^{2} T=n\left(4 / 3 \pi r^{3}\right)$ based on the volume equation of a square platelet shape and a round sphere, respectively. When the parameter $n=1$ or the same particle number, the correlation is shown in Figure 2 for different platelet thicknesses $(T=0.5,1.0$ or $2.0 \mathrm{~nm}$ ). For example, a platelet with the thickness of $1 \mathrm{~nm}$ and the dimension $100 \times 100 \mathrm{~nm}^{2}$ is approximately equivalent to a sphere of $13 \mathrm{~nm}$ in diameter $(r)$. When comparing a $10 \mathrm{~nm}$ diameter sphere with a $100 \times 100 \times 1 \mathrm{~nm}$ platelet, under this equation estimation, an individual platelet has a surface area equivalent to $n=2.4$ numbers of spherical particles. This approximation also indicates that the surface area of

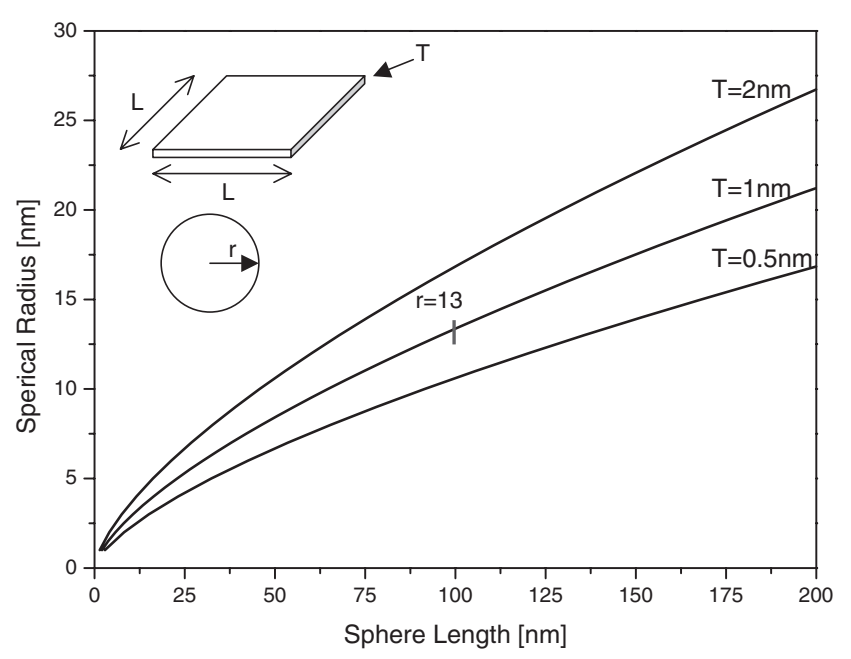

Figure 2. Correlation between the square platelet length $(L)$ and spherical radius $(r)$ in different platelet thicknesses according to $L^{2} T=4 / 3 \pi r^{3}$.

platelets $\left(100 \times 100 \times 1 \mathrm{~nm}^{3}\right)$ is about 7 times that of particles $(r=10 \mathrm{~nm})$ of the same weight. Hence, with a same weight of silicate addition in polymeric matrices, the surface interaction for the thin silicate platelets is much intense than that for silicate spheres.

\section{Homogeneous Dispersion of Silicates in Poly(oxypro- pylene)-triamine Curing Agent}

The second factor affecting the silicate interaction with organic polymers is the dispersion ability in the epoxy. The commercially available silica oxide particles with $\equiv \mathrm{Si}-\mathrm{OH}$ functionalities are hydrophilic and supplied as a $15 \mathrm{wt} \%$ suspension in water. Similarly, the random silicate platelets, prepared from the direct exfoliation of $\mathrm{Na}^{+}$-MMT, are also hydrophilic and dispersible in water due to the presence of sodium ions $\left(\equiv \mathrm{Si}-\mathrm{O}^{-} \mathrm{Na}^{+}\right)$on the platelet surface. Both silicate solutions were shown to have different geometric shapes under TEM examination. The silicate platelets have an irregular plate-like shape with the dimensions 80-100 nm, while the silicate oxides are aggregates with the dimension $10-50 \mathrm{~nm}$ (Figure 3). In order to minimize the incompatibility problem with the polymer matrix, a hydrophilic and water-soluble amine is chosen for dispersing the hydrophilic silicates. Both silicates were allowed to disperse in poly(oxypropylene)-triamine (Jeffamine ${ }^{\circledR}$ T403) and cured with the BPA diglycidyl ether resin. When the silicates are mixed with the T403 amine, both the silica sphere and platelets could be homogeneously dispersed, however, the two silicate materials exhibited different physical appearances. During the process of adding the water-suspended platelets into T403 amine and consequent water removal, the resultant silicate/ T403 dispersion became a light brown gel with an in- 



Figure 3. TEM micrograms of (a) silicate oxides $(15 \mathrm{wt} \%)$ and (b) silicate platelets suspension $(0.1 \mathrm{wt} \%)$ in water.

creased viscosity. In comparison, $6.0 \mathrm{wt} \%$ of silicate platelet addition caused the T403 amine to become gel-like semisolid in comparison with $25.0 \mathrm{wt} \%$ of silicate oxide addition to reach a gel point. The gel formation, which is more easily achieved by the platelet silicates than by silica oxides, implies a higher aspect ratio of these random silicate platelets and their increased interactions with the hydrophilic amine molecules.

\section{Properties of Epoxy Nanocomposites with Silicate Platelets and Silica Oxide Spheres}

As summarized in Table I, the silicate particles/ T403 dispersion consisting of $1-5 \mathrm{wt} \%$ fine silica oxide particles with an average $10 \mathrm{~nm}$ diameter, was prepared and then cured with epoxy materials. The selected epoxy system comprised of diglycidyl ether

Table I. Properties of amine-cured epoxy (T403/DGEBA) nanocomposites with silica oxides and silicate platelets

\begin{tabular}{lccccc}
\hline Silicates & $\begin{array}{c}\text { Loading } \\
(\%)\end{array}$ & $T_{\mathrm{g}}$ & $\begin{array}{c}\text { Thermal } \\
\text { Expansion } \\
\text { Coefficient } \\
(\alpha)\end{array}$ & $\begin{array}{c}\text { Hardness } \\
(\mathrm{H})\end{array}$ & $\begin{array}{c}\text { Transparency } \\
(\%)\end{array}$ \\
\hline None & 0 & 61 & 98 & 2 & 61 \\
$\mathrm{SiO}_{2}$ & 1 & 59 & 80 & $2-3$ & 54 \\
$\mathrm{SiO}_{2}$ & 5 & 58 & 72 & 4 & 45 \\
$\begin{array}{l}\text { Silicate } \\
\text { platelets }\end{array}$ & 0.5 & 73 & 90 & 4 & 55 \\
$\begin{array}{l}\text { Silicate } \\
\text { platelets }\end{array}$ & 1 & 64 & 95 & 5 & 48 \\
\hline
\end{tabular}

$T_{\mathrm{g}}$ : glass transition temperature.

Transparency: by UV-vis absorption. of BPA (DGEBA) at 1:1 molar ratio of epoxy/N-H. Upon adding the 1 and $5 \mathrm{wt} \%$ silicate particles, the cured polymer materials exhibited significantly enhanced hardness from the pristine $2 \mathrm{H}$ to $2-3 \mathrm{H}$ and $4 \mathrm{H}$, respectively. The nanocomposite was also characterized by using an optical UV-vis spectroscope. The material transparency was compared by determining the absorbance at $550 \mathrm{~nm}$ and was found to decrease from 61 to $45 \%$. The glass transition temperature was not significantly altered by the silicate oxide addition. By comparison, the hardness enhancement from $2 \mathrm{H}$ to $4 \mathrm{H}$ is achieved by adding only $0.5 \mathrm{wt} \%$ of silicate platelets. A measurement of $90 \%$ transparency was observed indicating a fine dispersion of the platelets without light interference. At this concentration, the material glass transition $\left(T_{\mathrm{g}}\right)$ has significantly increased from 61 to $73^{\circ} \mathrm{C}$. Furthermore, the thermal expansion coefficient $(\alpha)$ of the epoxy materials significantly decreased when mixed with the spherical particle compared to the platelet.

\section{Thermal Gravimetric Analysis (TGA) of Epoxy Nano- composites}

The thermal decomposition patterns of the silicate platelet- and sphere-added epoxies are analyzed by thermal gravimetric analysis. With an initial 5 wt \% weight loss, the decomposition temperatures were at 342,343 and $332{ }^{\circ} \mathrm{C}$ for platelet-, sphere- and noneadded epoxies, respectively (Figure 4). Although the initial decomposition temperatures are about $10^{\circ} \mathrm{C}$ different, the overall decomposition curves are similar. The use of nanoscaled silicates to stabilize the organic epoxy at $5 \mathrm{wt} \%$ addition level is not pronounced. 


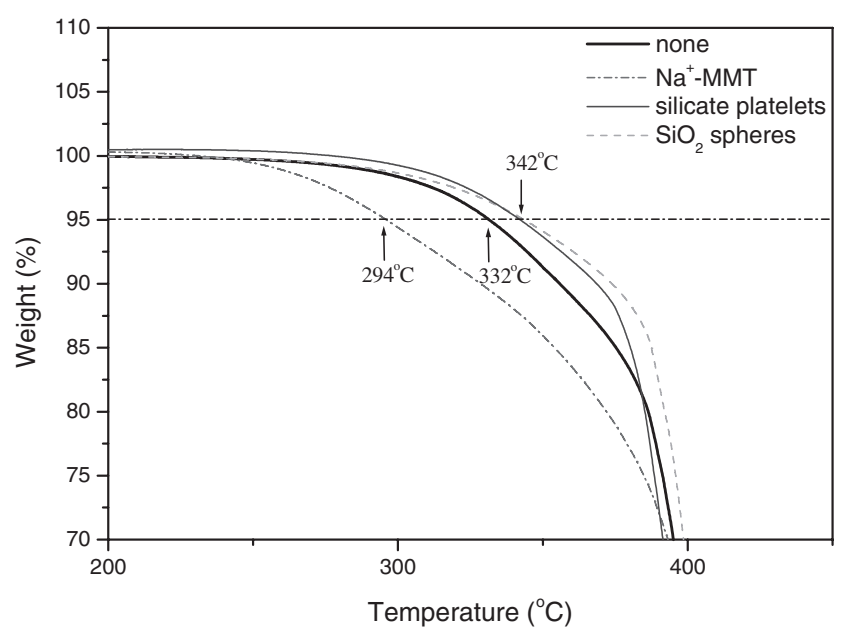

Figure 4. Thermal gravimetric analyses of epoxies with the pristine $\mathrm{Na}^{+}-\mathrm{MMT}$, silicate platelets and spheres (1 wt \%) at a heating rate of $10^{\circ} \mathrm{C} / \mathrm{min}$ in $\mathrm{N}_{2}$.

\section{SEM Morphologies of Epoxy Nanocomposites}

The SEM micrographs of the fracture surfaces of these epoxies reveal different degrees of phase separation phenomena (Figure 5). A smooth surface suggests poor adhesion between domain phases. There


are marked differences between the morphologies of the virgin epoxy and epoxies with silicate additions. For example, the 0.5 and $1.0 \mathrm{wt} \%$ silicate platelet dispersed epoxies have fracture surfaces with rougher irregularities compared to the non-additive epoxy (Figure 5a, d and e). When the silicate spheres ( $1 \mathrm{wt} \%)$ are added to the matrix, the inhomogeneous surface increase but less than that caused by silicate platelets. The adhesion effect is due to the highaspect-ratio effect of the platelets.

\section{Morphology of Lamellar Silicate Platelets in Epoxy Matrix}

The doping of finely dispersed silicate platelets in epoxy matrix has improved the material hardness and increase the $T_{\mathrm{g}}$ while maintaining the material's transparency. By TEM (Figure 6), the distribution of silicate platelets was observed to be in a bilayer orientation and forms stacks of lamellar platelets with one lamella over another. From the side view, the arrangement extended to a length of $200-500 \mathrm{~nm}$. The mechanism is conceptually explained in Scheme 1. The occurrences of unique transformations are attributed to the high aspect-ratio of the lamellar platelets as well
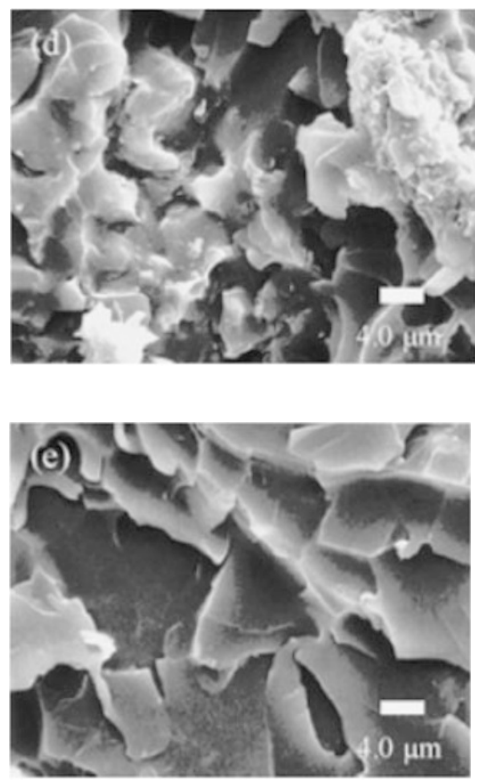

Figure 5. SEM micrograms of epoxy nanocomposite fracture surfaces (a) pristine epoxy and epoxies with the additions of (b) spheres $(1.0 \mathrm{wt} \%)(\mathrm{c})$ spheres $(5.0 \mathrm{wt} \%)(\mathrm{d})$ platelets $(0.5 \mathrm{wt} \%)$ (e) platelets $(1.0 \mathrm{wt} \%)$. 

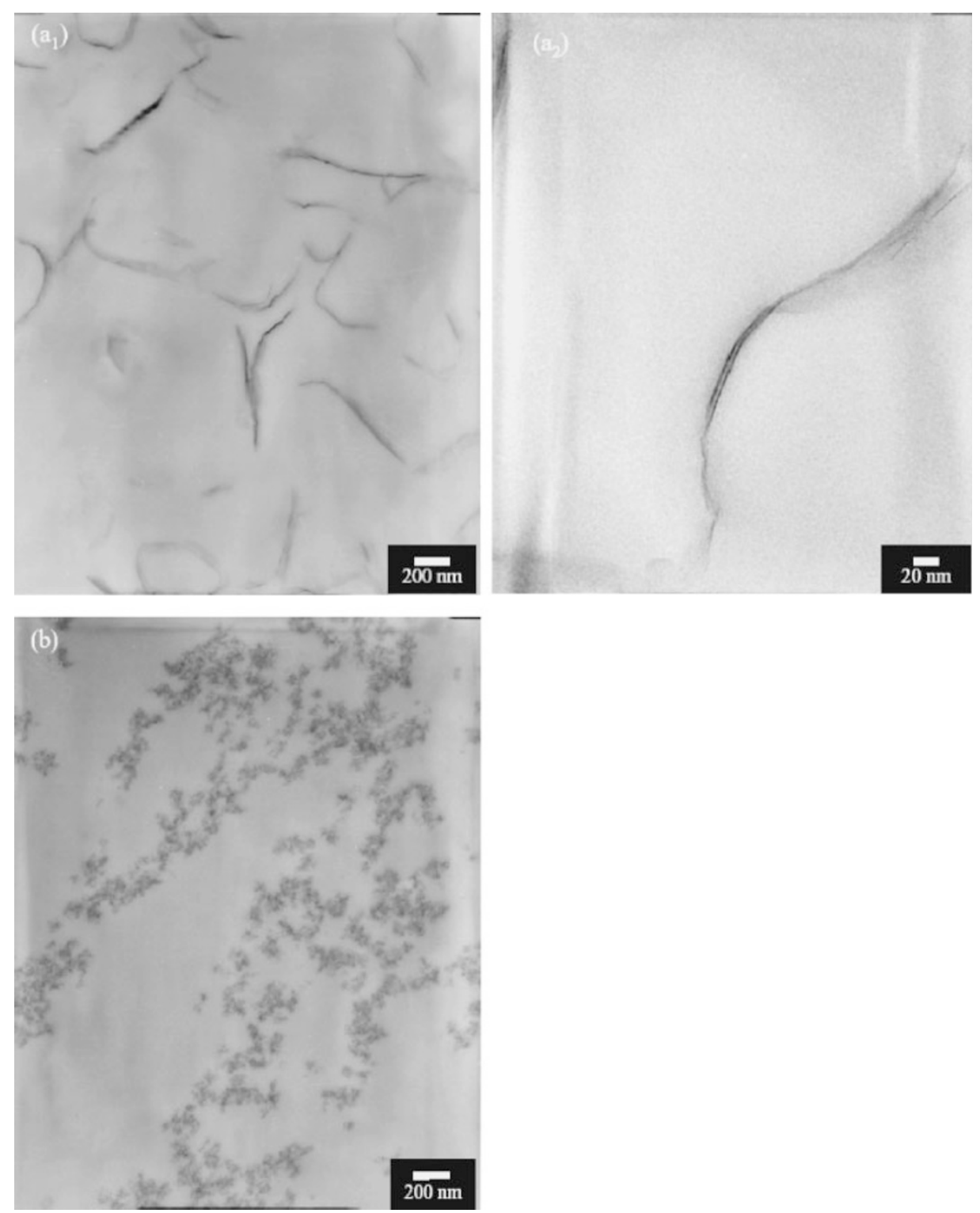

Figure 6. TEM micrograms of epoxy nanocomposites containing $\left(\mathrm{a}_{1}\right)$ silicate platelets $(1.0 \mathrm{wt} \%)\left(\mathrm{a}_{2}\right)$ bilayer orientation at a higher magnification and (c) silicate oxide particles $(3.0 \mathrm{wt} \%)$.

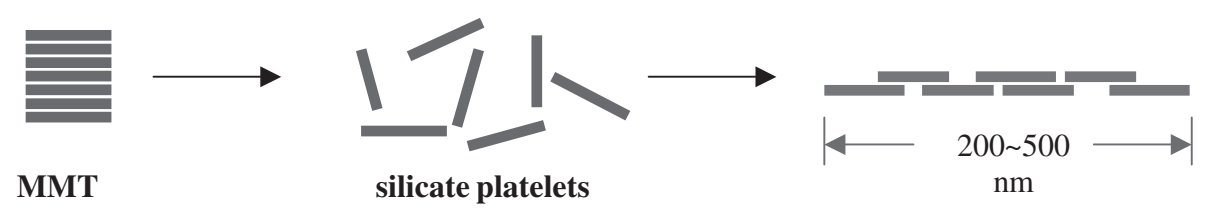

Scheme 1. Conceptual illustration of random silicate platelets self-piling into bilayer lamellae.

as the highly intense ionic character on the platelet surface. Compared with silicate platelets, the silicate particles in the epoxy matrix appear to be clustered and aggregated in groups (Figure 6b). The globular structure found may explain why the hardness and $T_{\mathrm{g}}$ are less affected when compared to the epoxy/ silicate hybrid.

\section{CONCLUSIONS}

By comparing the effect of different geometric shapes between silicate spheres and platelets on properties, we have determined that the high-aspect-ratio is a predominant factor for enhancing the material hardness. With $1.0 \mathrm{wt} \%$ silicate platelet loading, the 
cured epoxy exhibits $5 \mathrm{H}$ hardness, which is superior to that of silicate sphere additions $(2-3 \mathrm{H}$ by adding $1 \mathrm{wt} \%$ and $4 \mathrm{H}$ by $5 \mathrm{wt} \%$ ). The pronounced influence of platelet addition affects the material fracture surface, as shown by SEM. In addition, TEM studies revealed that the silicate platelets were finely dispersed in the polymer matrix in the form of a lamellar bilayer alignment with a $200-500 \mathrm{~nm}$ length. The lamellar piling in the polymer matrix and the intense silicate/ polymer interaction accounts for the improvements of these properties.

Acknowledgment. We acknowledge the financial support from National Science Council (NSC) of Taiwan and the assistants from the Regional Instruments Center at NCHU Analytical Electron Microscope.

\section{REFERENCES}

1. A. Okada and A. Usuki, Mater. Sci. Eng., C, 3, 109 (1995).

2. P. C. Lebaron, Z. Wang, and T. J. Pinnavaia, Appl. Clay Sci., 15, 11 (1999).

3. E. P. Giannelis, Adv. Mater., 8, 29 (1996).

4. Z. Wang and T. J. Pinnavaia, Chem. Mater., 10, 1820 (1998).

5. M. S. Wang and T. J. Pinnavaia, Chem. Mater., 6, 468 (1994).

6. T. Lan, P. D. Kaviratna, and T. J. Pinnavaia, J. Phys. Chem. Solids, 57, 1005 (1996).
7. H. Ishida, S. Campbell, and J. Blackwell, Chem. Mater., 12, 1260 (2000).

8. X. Fu and S. Qutubuddin, Polymer, 42, 807 (2001).

9. C. D. Muzny, B. D. Butler, H. J. M. Hanley, F. Tsvetkov, and D. G. Peiffer, Mater. Lett., 28, 379 (1996).

10. A. Akelah, N. S. El-Deen, A. Hiltner, E. Baer, and A. Moet, Mater. Lett., 22, 97 (1995).

11. A. Akelah and A. Moet, J. Appl. Polym. Sci., Appl. Polym. Symp., 55, 153 (1994).

12. M. Kato, A. Usuki, and A. Okada, J. Appl. Polym. Sci., 66, 1781 (1997).

13. H. L. Tyan, Y. C. Liu, and K. H. Wei, Chem. Mater., 11, 1942 (1999).

14. T. Agag and T. Takeichi, Polymer, 41, 7083 (2000).

15. M. Kawasumi, N. Hasegawa, M. Kato, A. Usuki, and A. Okada, Macromolecules, 30, 6333 (1997).

16. N. Hasegawa, H. Okamoto, M. Kawasumi, and A. Usuki, J. Appl. Polym. Sci., 74, 3359 (1999).

17. N. Ogata, S. Kawakage, and T. Ogihara, Polymer, 38, 5115 (1997).

18. J. W. Gilman, Appl. Clay Sci., 15, 31 (1999).

19. K. Jiří, Appl. Clay Sci., 10, 275 (1995).

20. J. C. Huang, Z. K. Zhu, J. Yin, X. F. Qian, and Y. Y. Sun, Polymer, 42, 873 (2001).

21. K. Yano, A. Usuki, A. Okada, T. Kurauchi, and O. Kamigaito, J. Polym. Sci., Part A: Polym. Chem., 31, 2493 (1993).

22. J. J. Lin, I. J. Cheng, R. Wang, and R. J. Lee, Macromolecules, 34, 8832 (2001)

23. J. J. Lin, C. C. Chu, and C. M. Tsai, Macromol. Rapid Commun., submitted. 